\title{
Differences in men and women suffering from CRSwNP and AERD in quality of life
}

\author{
Tina J. Bartosik ${ }^{1}$ - David T. Liu ${ }^{1}$ (1) - Nicholas J. Campion ${ }^{1} \cdot$ Sergio Villazala-Merino ${ }^{1}$ Stefan Janik ${ }^{1}$. Valerie Dahm ${ }^{1}$. \\ Christian A. Mueller $^{1}$. Erich Vyskocil ${ }^{1} \cdot$ Victoria Stanek $^{1} \cdot$ Tamara Quint $^{2} \cdot$ Christine Bangert $^{2} \cdot$ Julia Eckl-Dorna $^{1}{ }^{\mathbb{D}}$. \\ Sven Schneider ${ }^{1}$
}

Received: 10 September 2020 / Accepted: 3 October 2020 / Published online: 15 October 2020

(c) The Author(s) 2020

\begin{abstract}
Purpose While the overall impact of chronic rhinosinusitis (CRS) on patients' health is diverse, many affected individuals have a substantially impaired quality of life (QoL). The aim of this study was to evaluate the impact of sex-associated differences specifically in the subgroups of CRS with nasal polyps (CRSwNP) and aspirin-exacerbated respiratory disease (AERD) by assessing QoL parameters in women and men separately.

Methods In a retrospective single-center study, 59 patients with CRSwNP (39 males and 20 females) and 46 patients with AERD (18 males and 28 females) were included. Patient-reported outcome measures (PROM) evaluating QoL via the SinoNasal Outcome Test-20 German Adapted Version (SNOT-20 GAV) as well as the total polyp score (TPS) were analysed.

Results There was no significant difference in TPS $(p=0.5550)$ and total SNOT-20 GAV scores $(p=0.0726)$ between male or female patients with CRSwNP or AERD. Furthermore, no significant sex differences were found within disease groups regarding the subcategories of the SNOT-20 GAV items.
\end{abstract}

Conclusion Thus, quality of life is severely impaired in patients suffering from various forms of CRS regardless of their sex.

Keywords Chronic rhinosinusitis · Sex $\cdot$ Polyps $\cdot$ Aspirin-exacerbated respiratory disease $\cdot$ AERD $\cdot$ Samter's triad $\cdot$ SNOT$20 \mathrm{GAV}$

\section{Introduction}

Sex- and gender-related factors are present in a variety of diseases affecting the clinical presentation and treatment of these medical conditions, as well as the impact on patients' quality of life (QoL) [1]. Among others, sex-related aspects have not only been identified regarding genetics and hormones, but interestingly also when analysing epigenetics, immune function, aging including neurocognitive decline, vascular status as well as response to therapeutics and interaction with the health care system [2].

Chronic rhinosinusitis (CRS) is a common, yet diverse disease affecting $4-16 \%$ of the general population [3-5].

Julia Eckl-Dorna

julia.eckl-dorna@meduniwien.ac.at

1 Department of Otorhinolaryngology, Medical University of Vienna, Währinger Gürtel 18-20, 1090 Vienna, Austria

2 Department of Dermatology, Medical University of Vienna, Währinger Gürtel 18-20, 1090 Vienna, Austria
According to the recently published current version of the European Position Paper on Rhinosinusitis and Nasal Polyps (EPOS 2020), the condition should be classified into primary and secondary CRS. While the former is associated with generalized inflammation, the latter is caused by other underlying triggers including tumours, odontogenic reasons, or fungal growth. Primary CRS can be further sub-grouped as localized (unilateral) or diffuse (bilateral) disease and phenotypically grouped according to the absence or presence of nasal polyps. Phenotypes are determined by underlying type 2 (CRS with nasal polyps, CRSwNP) or non-type 2 inflammation (CRS without nasal polyps, CRSsNP) [6]. CRSwNP affects between 2.7 and $4.4 \%$ of the population and approximately $10 \%$ of these patients with nasal polyps suffer from aspirin-exacerbated respiratory disease (AERD) syndrome, which is characterized by the clinical triad of nasal polyps, bronchial asthma, and hypersensitivity to non-steroidal antiinflammatory drugs (NSAIDs) [6-8]. CRSwNP and AERD generate not only nasal symptoms but also impair quality of sleep, mood, cognition, and productivity [9]. 
Patient-reported outcome measures (PROMs) are important instruments to evaluate the subjective burden of disease. Sino-nasal outcome test-22 (SNOT-22) and the sino-nasal outcome test-20 German adapted version (SNOT-20 GAV) are valid, reliable, and widely used tools to specifically address CRS symptoms $[10,11]$. In this study, we focused on the comparison of patients suffering from CRS presenting with nasal polyps (CRSwNP) alone and those diagnosed with AERD.

Studies evaluating sex and gender specific impact on QoL of patients with CRSwNP and AERD are discordant. Furthermore, differences in men and women could also exist in specific phenotypes of CRS. Recently, a cluster analysis of SNOT-22 and VAS values regarding CRSwNP and CRSsNP patients according to gender was performed. Three clusters were identified: the first cluster comprised 37 female patients with CRS without nasal polyps (CRSsNP), the second cluster comprised 30 patients with CRS and NP (CRSwNP; 15 males and 15 females); and the third cluster had 30 male patients with CRS without NP (CRSsNP), indicating similarities in men and women diagnosed with CRSwNP but not CRSsNP patients [12]. With regards to sex as a factor influencing PROMs, higher SNOT-22 scores in patients suffering from CRS (with and without polyps) presenting for sinus surgery have been reported in women [13]. Elaborating on sex-specific differences in CRS, a higher prevalence of facial pain and headache was observed in female patients, whereas nasal obstruction was described more frequently in men [14]. In another study examining QoL of patients with CRS, worse QoL results were found among women in spite of similarities in objective disease measures, which were attributed to the increased prevalence of depression and AERD. When patients with depression or aspirin sensitivity were removed from the analysis, no statistically significant gender differences could be found [15].

Modern therapeutic strategies aim at personalized management of the individual patient. Therefore, knowledge of sex and sex-specific influences might be relevant to improve care for patients suffering from CRSwNP and AERD. Thus, the aim of this study was to clarify the impact of CRSwNP and AERD on patients' QoL in women and men in order to possibly adapt future therapies accordingly.

\section{Materials and methods}

\section{Study population}

The protocol was approved by the ethics committee of the Medical University of Vienna (EK 1630/2019) and the study was conducted according to the guidelines of the Declaration of Helsinki on Biomedical Research Involving Human Subjects. Patients with nasal polyps (aged 18 and older) presenting at the Department of Otorhinolaryngology and the Department of Dermatology at the Medical University of Vienna between 2017 and 2019 for further therapy were included and had to provide written informed consent. Subjects suffering from vasculitis, cystic fibrosis, odontogenic sinusitis, fungal sinusitis, sarcoidosis, and autoimmune disease (secondary CRS) were excluded.

During the visit, patients' sex and age were recorded. Patients were asked to complete a questionnaire including the SNOT-20 GAV and nasal polyposis was assessed via endoscopy by a trained otorhinolaryngologist. CRS was diagnosed based upon the criteria according to the EPOS 2020 [6]. Furthermore, the diagnosis of AERD was defined according to the European Academy of Allergy and Clinical Immunology (EAACI) position paper as the documented presence of nasal polyps and asthma, in addition to developing respiratory symptoms upon the ingestion of aspirin or other non-steroidal anti-inflammatory drugs (NSAID) [16]. If nasal polyps were present at any time in a patient's medical history, he or she was classified as CRSwNP. Consequently, patients' diagnoses remained CRSwNP or AERD even if nasal polyps were not present at the time of the study visit due to recent surgical removal.

\section{Outcome measures}

To grade the nasal polyp size by nasal endoscopy, the total polyp score (TPS) system was used [17]. Both sides of the nasal cavity were separately assessed and scored based on polyp size, resulting in scores of $0-4(0=$ no polyps, $1=$ small polyps in the middle meatus not reaching below the inferior border of the middle turbinate, $2=$ polyps reaching below the lower border of the middle turbinate, $3=$ large polyps reaching the lower border of the inferior turbinate or polyps medial to the middle turbinate, $4=$ large polyps causing complete obstruction of the inferior nasal cavity) [18]. The sum of both nostril scores was considered as the TPS.

The SNOT-20 GAV, was used to record QoL $[10,19]$. SNOT-20 GAV is a reliable, validated and sensitive German instrument for measuring health-related QOL in patients with CRS [20]. SNOT- 20 GAV differs from the widely used version in English SNOT-22 as the items "need to blow nose", "lack of good night's sleep" and "fatigue" are not represented in SNOT-20 GAV, but additional item "need to clear throat/dry throat" is included. The parameters of the test are graded from 0 to $5(0=$ no problem, $1=$ very mild problem, $2=$ mild or slight problem, $3=$ moderate problem, $4=$ severe problem, $5=$ problem as bad as can be). Moreover, the 20 questions were summarized in four subcategories: nasal symptoms ("nasal blockage", "sneezing", "runny nose", "post-nasal discharge", "thick nasal discharge", "need to clear throat/dry throat", "cough", "sense of smell"), otologic symptoms ("ear congestion”, "ear pain", "dizziness", 
"facial pain/pressure"), sleep symptoms ("difficulty falling asleep", "waking up at night", "fatigued or tired during the day", "reduced productivity", "reduced concentration", "frustration, restlessness, irritability") and emotional symptoms ("sad", "embarrassed"). Each category, as well as each symptom, was analysed separately.

\section{Statistical analysis}

Study data were collected and managed using Microsoft Excel for iOS 16.25 (Microsoft Corporation, Washington, USA). GraphPad Prism 8.4.2 (GraphPad Software, Inc., La Jolla, San Diego, CA, USA) was used for statistical analysis and visualization of the results. Normality of data was tested using Kolmogorov-Smirnov test with Dallal WilkinsonLille being used for calculation of the $p$ value.

The patient characteristics like age, TPS, number of previous surgeries, and the SNOT score were described as mean ( \pm standard deviation). Welch's ANOVA was used to compare group differences and followed by Dunnett's T3 multiple comparisons test.

A $p$ value of $<0.05$ was required for statistical significance.

\section{Results}

\section{Patient characteristics}

A total of 105 patients with nasal polyps presenting to the outpatient clinic of the Department of Otorhinolaryngology or the Department of Dermatology at the Medical University of Vienna were included in this study. Among patients suffering from isolated CRSwNP, 66\% $(n=39)$ were male and $34 \%(n=20)$ were female. $39 \%(n=18)$ of the AERD patients were male and $61 \%(n=28)$ female. Patient details are depicted in Table 1 and Fig. 1 a.

The mean age of patients was 46.6 years and no statistically significant differences in age were observed between the stratified male and female groups (Fig. 1b).

\section{TPS and history of surgeries in patients suffering from CRSwNP or AERD according to sex}

In the group of patients suffering from CRSwNP alone, the mean TPS was $3.87(\mathrm{SD}=2.27)$ in male and $3.40(\mathrm{SD}=1.57)$ in female patients (Table 1; Fig. 2a). Male AERD patients scored a mean TPS of $4.44(\mathrm{SD}=2.99)$ and females a mean of $3.54(\mathrm{SD}=2.67)$. No significant differences in the four subgroups were observed (Welch's ANOVA $p=0.5550$ ).

No significant differences in female and male patients according the number of previous surgeries were observed within the disease groups (Dunnett's T3 multiple comparison test CRSwNP male vs. female $p>0.9999$ and AERD male vs. female $p=0.2286$ ). Of the CRSwNP patients, $44.93 \%(n=33)$ had prior surgery compared to $91.30 \%$ $(n=42)$ in the AERD group. The mean number of previous surgeries was $0.82(\mathrm{SD}=0.95)$ in male $\mathrm{CRSwNP}$ patients, $0.75(\mathrm{SD}=0.91)$ in female with CRSwNP, $3.72(\mathrm{SD}=3.32)$ in male AERD patients, and $1.96(\mathrm{SD}=1.37)$ in female AERD patients (Table 1). On the other hand, significant differences in pairwise comparisons using Dunnett's T3 multiple comparison test were shown between CRSwNP male and AERD male ( $p=0.0108)$, CRSwNP male and AERD female $(p=0.0031)$, CRSwNP female and AERD male $(p=0.0094)$, and CRSwNP female and AERD female $(p=0.0042)$.

\section{SNOT-20 GAV score in male and female patients suffering from CRSwNP or AERD}

In the group of patients suffering from CRSwNP, the mean SNOT-20 GAV score was $33.91(\mathrm{SD}=15.74)$ in male and 40.40 ( $S D=20.01)$ in female patients. Male AERD patients scored a mean SNOT-20 GAV score of $47.56(\mathrm{SD}=21.14)$ and females a mean of $41.79(\mathrm{SD}=18.48)$.

No significant differences between male and female patients were observed (Table 1; Fig. 3a). However, when analyzing the four categories of the SNOT-nasal, otologic, sleep and emotional symptoms - male patients with AERD suffered significantly more from "nasal symptoms" as compared to male patients with CRSwNP (Dunnett's T3 multiple comparison test $p=0.0121$ ).

Table 1 Patients characteristics

\begin{tabular}{|c|c|c|c|c|c|c|c|c|}
\hline & \multicolumn{2}{|c|}{$\begin{array}{l}\text { CRSwNP male } \\
(n=39)\end{array}$} & \multicolumn{2}{|c|}{$\begin{array}{l}\text { CRSwNP female } \\
(n=20)\end{array}$} & \multicolumn{2}{|c|}{ AERD male $(n=18)$} & \multicolumn{2}{|c|}{$\begin{array}{l}\text { AERD female } \\
(n=28)\end{array}$} \\
\hline & Mean & SD & Mean & SD & Mean & SD & Mean & SD \\
\hline Age & 45.36 & 13.41 & 51.10 & 13.01 & 46.61 & 15.00 & 43.36 & 12.69 \\
\hline TPS & 3.87 & 2.27 & 3.40 & 1.57 & 4.44 & 2.99 & 3.54 & 2.67 \\
\hline SNOT score & 33.91 & 15.74 & 40.40 & 20.01 & 47.56 & 21.14 & 41.79 & 18.48 \\
\hline No. of surgeries & 0.82 & 0.95 & 0.75 & 0.91 & 3.72 & 3.32 & 1.96 & 1.37 \\
\hline
\end{tabular}


Fig. 1 Sex and age distribution in patients suffering from chronic rhinosinusitis with nasal polyps (CRSwNP, green) or aspirin-exacerbated respiratory disease (AERD, red). a Sex distribution in patients with CRSwNP or AERD. b Average age ( $y$-axis, in years) in the two patient groups divided by sex $(x$-axis). Bars represent mean values with standard deviation (SD). Filled bars represent male patients, striped bars represent female patients. No significant differences were observed between the groups (Welch's ANOVA $p=0.2591) . n s$ not significant

A

\section{CRSwNP}

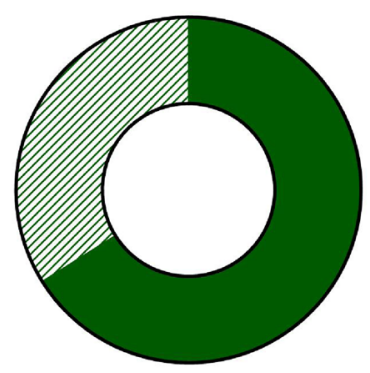

\section{AERD}
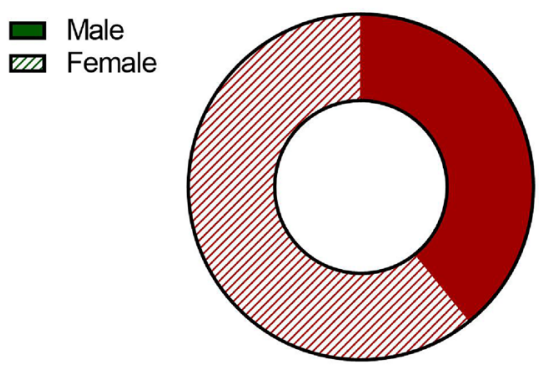

$\square$ Male ए] Female
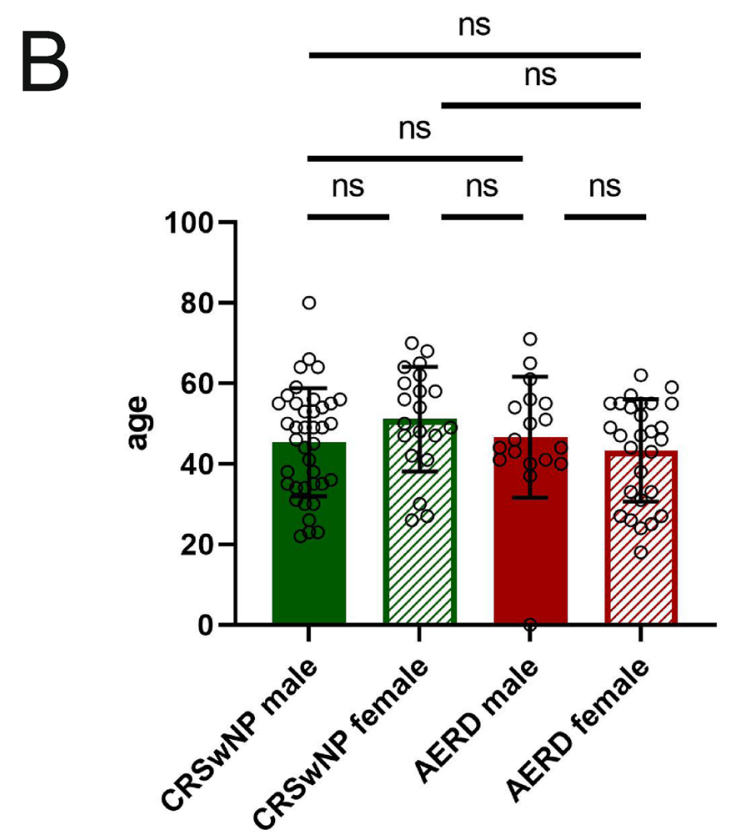

\section{Single item scores with significant differences between male and female patients suffering from CRSwNP or AERD}

Next, we analysed the single items of the SNOT-GAV 20 stratified by disease and sex and found no signifcant sex differences within disease groups. However, in the category nasal symptoms, male patients with AERD suffered more from the items post-nasal (Welch's ANOVA $p=0.0162$; Dunnett's T3 multiple comparison test $p=0.0094)$ and thick nasal discharge (Welch's ANOVA $p=0.0027$; Dunnett's T3 multiple comparison test $p=0.0017)$ as compared to their CRSwNP counterparts (Fig. 4a, b). Both male and female patients with AERD scored higher in the item 'Difficulty to feel 'smells' or "tastes" than male patients with CRSwNP (Fig. 4c; Welch's ANOVA $p=0.0049$; Dunnett's T3 multiple comparison test CRSwNP male vs. AERD male $p=0.0159$, CRSwNP male vs. AERD female $p=0.0056$ ). Females with AERD showed significant differences as compared to CRSwNP male patients with regard to the items "Dizziness or Vertigo" (category otologic symptoms; Welch's ANOVA $p=0.0076$; Dunnett's T3 multiple comparison test CRSwNP male vs. AERD female $p=0.0456$ )(Fig. 4d) and "Frustrated, restless or irritated" (category emotional symptoms; Welch's ANOVA $p=0.0004$; Dunnett's T3 multiple comparison test CRSwNP male vs. AERD female $p=0.0003$ ) (Fig. 4e). In all the other items, no significant differences between the four groups were observed (data not shown). 
Fig. 2 Total polyp score (TPS) and previous surgeries in male (filled bars) and female (striped bars) patients suffering from chronic rhinosinusitis with nasal polyps alone (CRSwNP, green) or aspirinexacerbated respiratory disease (AERD, red). a TPS (y-axis) in the two patient groups according to sex ( $x$-axis). Bars represent mean values with standard deviation (SD). No significant differences were observed between the groups (Welch's ANOVA $p=0.5550$ ). b Percentage of patients who had previous surgery ( $y$-axis) in the two patient groups according to sex ( $x$-axis). Dark colors represent no prior surgeries whereas light colors signal previous surgeries. c Average number of previous surgeries per patient group. Significant differences between patient groups were observed (Welch's ANOVA: $p<0.0001$ ), significant differences in pairwise comparisons using Dunnett's T3 multiple comparison test were shown between CRSwNP male and AERD male $(p=0.0108)$, CRSwNP male and AERD female $(p=0.0031)$, CRSwNP female and AERD male $(p=0.0094)$, and CRSwNP female and AERD female $(p=0.0042) . * p \leq 0.05, * * p \leq 0.01, * * * p \leq 0.001, * * * * p \leq 0.0001, n s$ not significant

\section{Discussion}

In this study, we examined the association of sex and QoL in patients suffering from CRSwNP and AERD. So far, aspects of gender and sex differences in these entities have been poorly investigated. We conducted this study with a large cohort of AERD $(n=46)$ and CRSwNP patients $(n=59)$. In summary, we found no significant difference in TPS and total SNOT-20 GAV scores between male or female patients with CRSwNP or AERD.

With the aim of achieving precision medicine, aspects of biological sex and the psychosocial aspects of a patient's gender identity are receiving growing attention in various disease states [21-23]. A wide range of factors including genetics, epigenetics, hormones, immune function, and behaviour might be influenced by patients' sex and gender identity [2]. CRS is a highly prevalent disease and especially phenotypes with nasal polyps and AERD can significantly impair patients' QoL [9]. Although the impact of sex in CRSwNP and AERD is not yet fully elucidated, studies evaluating e.g. the nasal microbiome in patients with CRS suggest differences in microbial colonization in women and men [24]. Furthermore, sex and gender specific differences might differ in CRS phenotypes like CRsNP, CRSwNP patients and patients suffering from AERD. A recently published cluster analysis evaluating QoL of CRS patients displayed three groups with similar symptoms. One cluster comprised women with CRSsNP, another cluster grouped men with CRSwNP and the third cluster represented women and men diagnosed with CRSwNP [12].

The QoL and impairment of olfactory function in AERD patients has been confirmed by previous research $[25,26]$, but interestingly, AERD and especially severe cases are more prevalent amongst women [27]. It has been reported that women and men can perceive and report symptoms and burden of disease differently [28]. It was
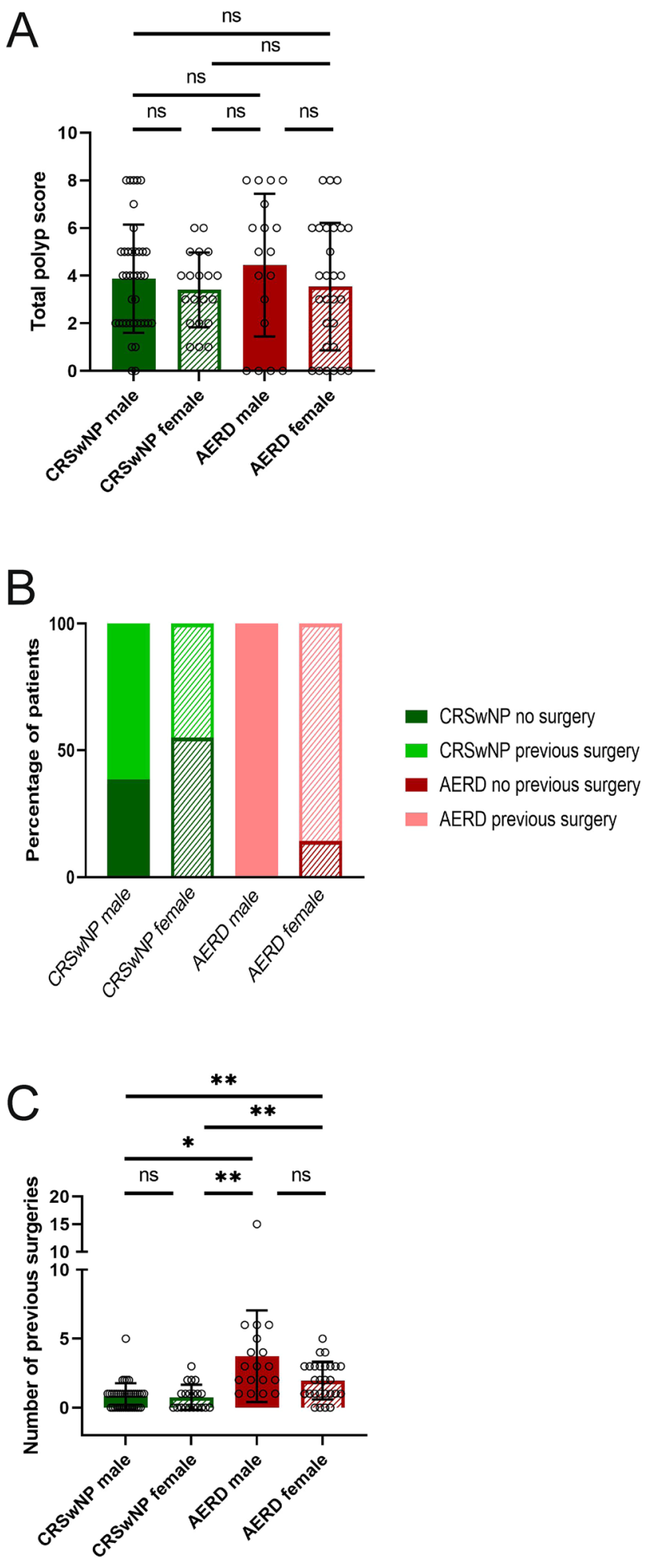

considered that women are more likely to report their symptoms and give a worse assessment of their health. However, the extent of the sex differences in health also vary according to the particular symptom or condition and to the phase of life [29]. It could, therefore, be hypothesized that studies in CRS, which assess the subjective 
Fig. 3 Sino-Nasal Outcome Test-20 German Adapted Version (SNOT-20 GAV) score in patients suffering from chronic rhinosinusitis with nasal polyps (CRSwNP, green) or aspirinexacerbated respiratory disease (AERD, red). a SNOT-20

GAV score ( $y$-axis) in the two patient groups displayed by sex ( $x$-axis). No significant differences were observed between the groups (Welch's ANOVA $p=0.0726$ ). b SNOT-20 GAV grouped into the four categories "nasal symptoms", "otologic symptoms", "sleep symptoms", and "emotional symptoms".

No significant differences were observed except for between the male CRSwNP and AERD patients in the category of "nasal symptoms" (Welch's ANOVA $p=0.0200$; Dunnett's T3 multiple comparison test $p=0.0121$ ). Filled bars represent male patients, striped bars female patients. Bars represent mean values with standard deviation (SD). Significance is displayed in figures $* p \leq 0.05$, $* * p \leq 0.01, * * * p \leq 0.001$, $* * * * p \leq 0.0001, n s$ not significant
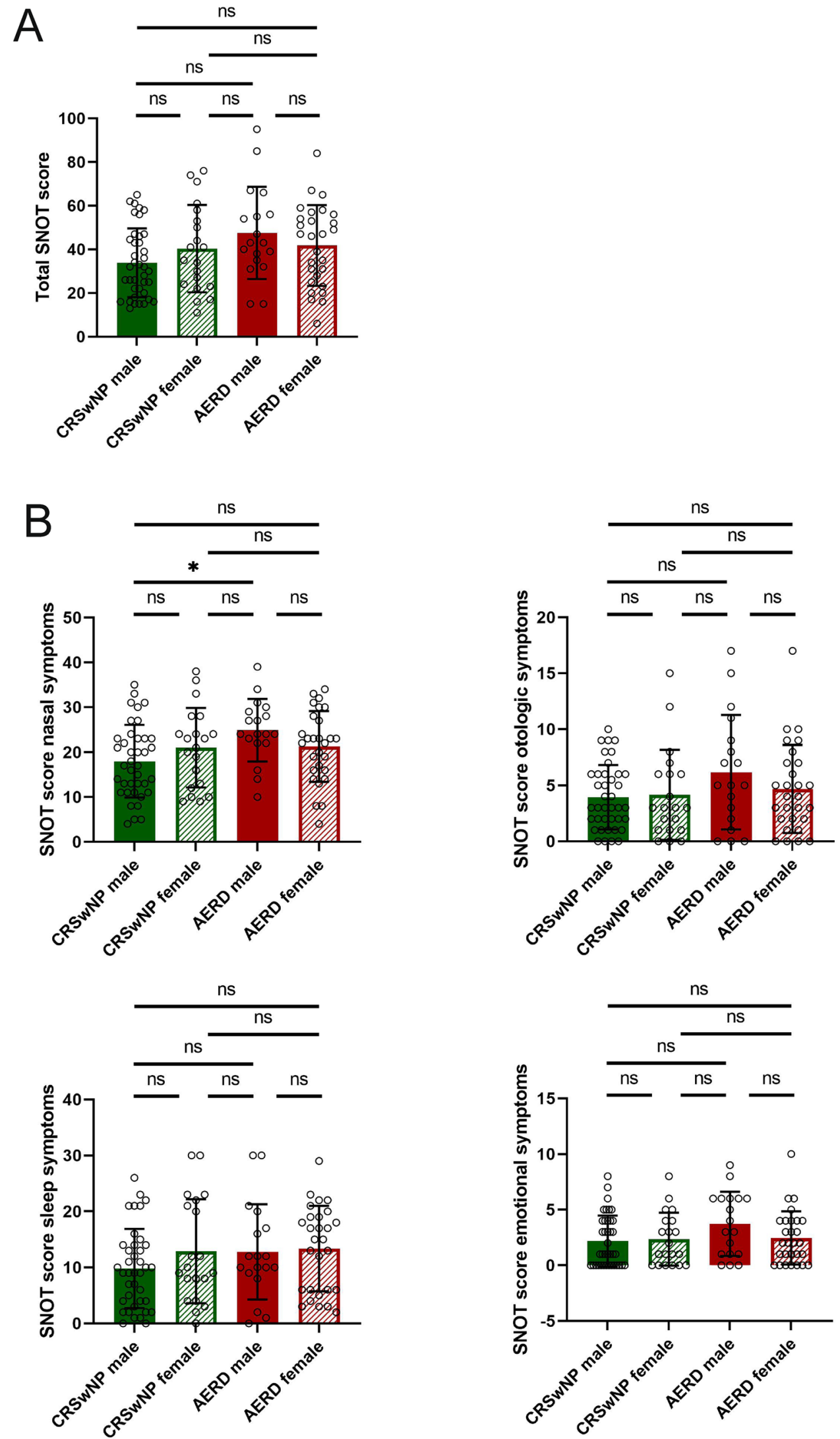

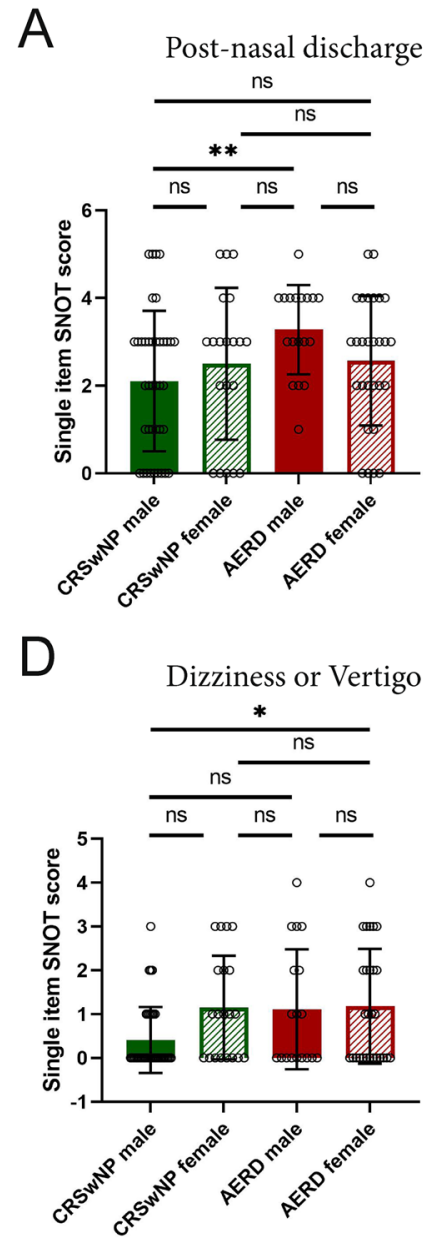

B

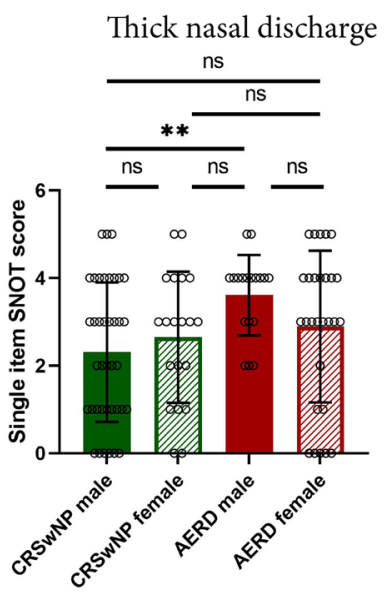

$E$

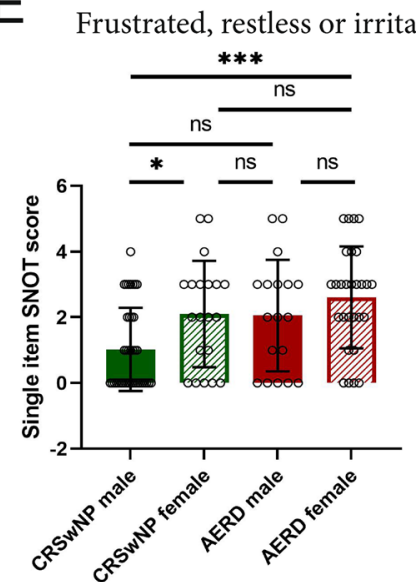

C

Difficulty to feel „smells" or „tastes“

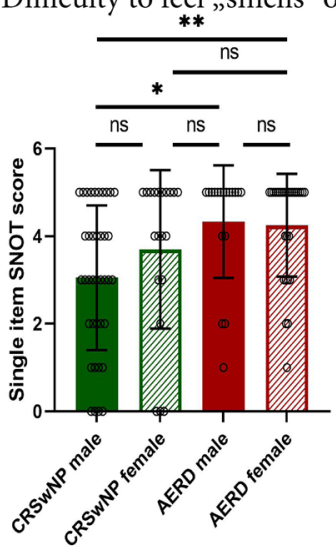

Fig. 4 Single item score of SNOT-20 GAV comparison in patients suffering from chronic rhinosinusitis with nasal polyps (CRSwNP, green) and aspirin-exacerbated respiratory disease (AERD, red) (A-E) SNOT-20 GAV score ( $y$-axis) in the two patient groups divided by sex $(x$-axis, male $=$ filled bars, female $=$ striped bars $)$ for the following items: a Post-nasal discharge (Welch's ANOVA $p=0.0162$ ). b Thick nasal discharge (Welch's ANOVA $p=0.0027$ ). $\mathbf{c}$ Difficulty to

complaints by the participants, would show a lower QoL among female patients.

Interestingly, we did not observe any significant differences in QoL between male and female patients diagnosed with CRSwNP and AERD. Similarly, Mendolia-Loffredo et al. also found no difference in disease-specific healthrelated QoL between sexes of patients with CRS [15, 30]. In this study analysis of the whole cohort revealed that females seemed to score worse than males on QoL scores. However, when further analysing the data, the authors noted that the poorer outcome was caused by the higher prevalence of acetylsalicylic acid intolerance and depression among women. Consequently, differences in QoL according to sex were eliminated after exclusion of patients with these comorbidities [15]. These results are in line with our observation where we could not find any major differences in QoL scores feel "smells" or "tastes" (Welch's ANOVA $p=0.0049$ ). d Dizziness or Vertigo (Welch's ANOVA $p=0.0076$ ). e frustration, restlessness or irritation" (Welch's ANOVA $p=0.0004$ ). Bars represent mean values with standard deviation (SD). Significance is displayed in figures $* p \leq 0.05, * * p \leq 0.01, * * * p \leq 0.001, * * * * p \leq 0.0001$, ns not significant

between male and females regardless whether they suffered from CRSwNP or AERD. It is, therefore, conceivable that the influence of sex is restricted primarily to the general QoL and that symptoms such as migraine headache, which are more common among women, and factors such as difference in anatomic size and hormone increases the prevalence of CRS in women compared to men as suggested by Ference et al. [30].

In the analysis of SNOT-20 GAV single items, we found the highest scores and significant differences in the symptom parameters "thick nasal discharge", "post-nasal discharge", and "difficulty to smell or taste" between the groups with CRSwNP and AERD in male patients only. In line with these results, Schneider et al. also observed higher SNOT-20 GAV scores in AERD patients as compared to CRSwNP especially in the category nasal 
symptoms [9]. The fact that they only observed significant differences between those two groups with regard to the single item "difficulty to smell or taste" but not in the other two above-mentioned items may be due to the fact that no stratification according to gender was performed.

Here, we specifically analysed patients suffering from CRSwNP or AERD in a retrospective evaluation of questionnaires. However, with regard to CRS (comprising both CRSsNP and CRSwNP), Baumann et al. showed in a prospective study that women preoperatively performed worse with regard to QoL score as compared to men [31, 32]. Interestingly, postoperatively no difference between the two gender groups was observed. Similar results were shown by Lal et al. who investigated gender-specific differences in CRS patients electing endoscopic sinus surgery in a retrospective review and reported more problems with postnasal drainage, embarrassment, and facial pain in women preoperatively. In their postoperative analysis, men and women showed similar symptom scores [13]. Since our study was a retrospective evaluation of the questionnaires and independent of the time or number of surgeries, this could be a reason for the non-significant differences in symptoms between the groups. Another limitation of our study is the relatively small sample size of patients with CRSwNP in comparison to other studies. However, it needs to be mentioned that other studies did not split the subgroups of chronic sinusitis with nasal polyps and thus failed to investigate sex-specific differences depending on the presence of polyps and subtypes. By being the first to differentiate the subgroups, this study contributed to understanding differences according to sex in patients suffering from various forms of nasal polyposis.

Future studies should aim at prospectively investigating the differences in sex-specific complaints of patients with CRSwNP and AERD before and after surgical intervention. In light of current developments it would also be interesting to assess patients' QoL before and after therapy with monoclonal antibodies targeting IgE, interleukins and interleukin receptors, which have shown promising effects in patients suffering from CRSwNP [33].

\section{Conclusion}

This is the first study assessing the differences in QoL between CRSwNP and a large cohort of AERD patients in relation to sex. The aim of this study was to clarify the impact of these diseases on patients' QoL in men and women to possibly highlight important differences to be taken into account for therapeutic treatment. We did not observe significant differences in TPS or QoL between the study groups.
Funding Open access funding provided by Medical University of Vienna. This research received no specific grant from any funding agency in the public, commercial, or not-for-profit sectors.

\section{Compliance with ethical standards}

Conflict of interest The authors declare no conflict of interest.

Ethics approval The protocol was approved by the ethics committee of the Medical University of Vienna (EK 1630/2019) and the study was conducted according to the guidelines of the Declaration of Helsinki on Biomedical Research Involving Human Subjects.

Availability of data and material Patients presenting at the Department of Otorhinolaryngology and the Department of Dermatology at the Medical University of Vienna between 2017 and 2019 had to provide written informed consent.

Open Access This article is licensed under a Creative Commons Attribution 4.0 International License, which permits use, sharing, adaptation, distribution and reproduction in any medium or format, as long as you give appropriate credit to the original author(s) and the source, provide a link to the Creative Commons licence, and indicate if changes were made. The images or other third party material in this article are included in the article's Creative Commons licence, unless indicated otherwise in a credit line to the material. If material is not included in the article's Creative Commons licence and your intended use is not permitted by statutory regulation or exceeds the permitted use, you will need to obtain permission directly from the copyright holder. To view a copy of this licence, visit http://creativecommons.org/licenses/by/4.0/.

\section{References}

1. Regitz-Zagrosek V (2012) Sex and gender differences in health. EMBO Rep 13:596-603. https://doi.org/10.1038/embor.2012.87

2. Bartz D, Chitnis T, Kaiser UB et al (2020) Clinical advances in sex- and gender-informed medicine to improve the health of all: a review. JAMA Intern Med 180:574-583. https://doi.org/10.1001/ jamainternmed.2019.7194

3. DeConde AS, Soler ZM (2016) Chronic rhinosinusitis: epidemiology and burden of disease. Am J Rhinol Allergy 30:134-139. https://doi.org/10.2500/ajra.2016.30.4297

4. Hastan D, Fokkens WJ, Bachert C et al (2011) Chronic rhinosinusitis in Europe-an underestimated disease. A GA2LEN study. Allergy 66:1216-1223. https://doi.org/10.111 1/j.1398-9995.2011.02646.x

5. de Loos DD, Lourijsen ES, Wildeman MAM et al (2019) Prevalence of chronic rhinosinusitis in the general population based on sinus radiology and symptomatology. J Allergy ClinImmunol 143:1207-1214. https://doi.org/10.1016/j.jaci.2018.12.986

6. Fokkens WJ, Lund VJ, Hopkins C et al (2020) European position paper on rhinosinusitis and nasal polyps 2020. Rhinology 58:1-464. https://doi.org/10.4193/Rhin20.600

7. Bachert C, Akdis CA (2016) Phenotypes and emerging endotypes of chronic rhinosinusitis. J Allergy ClinImmunolPract 4:621-628. https://doi.org/10.1016/j.jaip.2016.05.004

8. Rajan JP, Wineinger NE, Stevenson DD, White AA (2015) Prevalence of aspirin-exacerbated respiratory disease among asthmatic patients: a meta-analysis of the literature. J Allergy ClinImmunol 135:676-681.e1. https://doi.org/10.1016/j.jaci.2014.08.020 
9. Schneider S, Campion NJ, Villazala-Merino S et al (2020) Associations between the quality of life and nasal polyp size in patients suffering from chronic rhinosinusitis without nasal polyps, with nasal polyps or aspirin-exacerbated respiratory disease. J Clin Med 9:925. https://doi.org/10.3390/jcm9040925

10. Baumann I, Plinkert PK, Maddalena HD (2008) Entwicklungeinerbewertungsskalafür den sino-nasal outcome test-20 german adapted version (SNOT-20 GAV). HNO 56:784-788. https://doi. org/10.1007/s00106-007-1606-9

11. Morley A, d., Sharp H r. (2006) A review of sinonasal outcome scoring systems - which is best? ClinOtolaryngol 31:103-109. https://doi.org/10.1111/j.1749-4486.2006.01155.x

12. Jurlin L, Gregurić T, Baudoin T et al (2019) Cluster analysis of chronic rhinosinusitis suggests gender-based differences. ORL J OtorhinolaryngolRelat Spec 81(1):1-9. https://doi. org/10.1159/000492966

13. Lal D, Golisch KB, Elwell ZA et al (2016) Gender-specific analysis of outcomes from endoscopic sinus surgery for chronic rhinosinusitis. Int Forum Allergy Rhinol 6:896-905. https://doi. org/10.1002/alr.21773

14. Busaba NY, Sin H-J, Salman SD (2008) Impact of gender on clinical presentation of chronic rhinosinusitis with and without polyposis. J LaryngolOtol 122:1180-1184. https://doi.org/10.1017/ S0022215107001302

15. Mendolia-Loffredo S, Laud PW, Sparapani R et al (2006) Sex differences in outcomes of sinus surgery. Laryngoscope 116:11991203. https://doi.org/10.1097/01.mlg.0000224575.12945.90

16. Kowalski ML, Agache I, Bavbek S et al (2019) Diagnosis and management of NSAID-exacerbated respiratory disease (N-ERD)—a EAACI position paper. Allergy 74:28-39. https:// doi.org/10.1111/all.13599

17. Meltzer EO, Hamilos DL, Hadley JA et al (2006) Rhinosinusitis: Developing guidance for clinical trials. J Allergy ClinImmunol 118:S17-S61. https://doi.org/10.1016/j.jaci.2006.09.005

18. Gevaert P, Calus L, Zele TV et al (2013) Omalizumab is effective in allergic and nonallergic patients with nasal polyps and asthma. J Allergy ClinImmunol 131:110-116.e1. https://doi.org/10.1016/j. jaci.2012.07.047

19. Piccirillo JF, Merritt MG, Richards ML (2002) Psychometric and clinimetric validity of the 20-Item Sino-Nasal Outcome Test (Snot-20). Otolaryngol Neck Surg 126:41-47. https://doi. org/10.1067/mhn.2002.121022

20. Baumann I, Blumenstock G, DeMaddalena H et al (2007) LebensqualitätbeiPatienten mit chronischer Rhinosinusitis. HNO 55:42-47. https://doi.org/10.1007/s00106-005-1347-6

21. Gemmati D, Varani K, Bramanti B et al (2020) "Bridging the Gap" everything that could have been avoided if we had applied gender medicine, pharmacogenetics and personalized medicine in the gender-omics and sex-omics era. Int J Mol Sci 21:296. https:// doi.org/10.3390/ijms21010296
22. Baetta R, Pontremoli M, Martinez Fernandez A et al (2018) Proteomics in cardiovascular diseases: unveiling sex and gender differences in the era of precision medicine. J Proteomics 173:62-76. https://doi.org/10.1016/j.jprot.2017.11.012

23. Ulrich BC, Guibert $\mathrm{N}$ (2018) Immunotherapy efficacy and gender: discovery in precision medicine. Transl Lung Cancer Res 7:S211S213. https://doi.org/10.21037/tlcr.2018.08.05

24. Golan Y, Gavriel H, Lazarovich T, Eviatar E (2017) Gender differences in the bacteriology of rhinosinusitis. Eur Arch Otorhinolaryngol 274:2803-2807. https://doi.org/10.1007/s0040 5-017-4560-5

25. Jang DW, Comer BT, Lachanas VA, Kountakis SE (2014) Aspirin sensitivity does not compromise quality-of-life outcomes in patients with samter's triad. Laryngoscope 124:34-37. https://doi. org/10.1002/lary.24220

26. Katotomichelakis M, Riga M, Davris S et al (2009) Allergic rhinitis and aspirin-exacerbated respiratory disease as predictors of the olfactory outcome after endoscopic sinus surgery. Am J Rhinol Allergy 23:348-353. https://doi.org/10.2500/ajra.2009.23.3325

27. Kennedy JL, Stoner AN, Borish L (2016) Aspirin-exacerbated respiratory disease: prevalence, diagnosis, treatment, and considerations for the future. Am J Rhinol Allergy 30:407-413. https:// doi.org/10.2500/ajra.2016.30.4370

28. Bartley EJ, Fillingim RB (2013) Sex differences in pain: a brief review of clinical and experimental findings. BJA Br J Anaesth 111:52-58. https://doi.org/10.1093/bja/aet127

29. Macintyre S, Hunt K, Sweeting H (1996) Gender differences in health: are things really as simple as they seem? Soc Sci Med 42:617-624. https://doi.org/10.1016/0277-9536(95)00335-5

30. Ference EH, Tan BK, Hulse KE et al (2015) Commentary on gender differences in prevalence, treatment, and quality of life of patients with chronic rhinosinusitis. Allergy Rhinol 6:e82-e88. https://doi.org/10.2500/ar.2015.6.0120

31. Baumann I, Blumenstock G (2005) Impact of gender on general health-related quality of life in patients with chronic sinusitis. Am J Rhinol 19:282-287

32. Baumann I, Blumenstock G, Zalaman IM et al (2007) Impact of gender, age, and comorbidities on quality of life in patients with chronic rhinosinusitis. Rhinology 45:268-272

33. Ren L, Zhang N, Zhang L, Bachert C (2019) Biologics for the treatment of chronic rhinosinusitis with nasal polyps - state of the art. World Allergy Organ J 12:100050. https://doi.org/10.1016/j. waojou.2019.100050

Publisher's Note Springer Nature remains neutral with regard to jurisdictional claims in published maps and institutional affiliations. 